VOL. 65 (2002) [371-383]

\title{
FRÉCHET ALGEBRAS WITH A LAURENT SERIES GENERATOR AND THE ANNULUS ALGEBRAS
}

\author{
S.J. Bhatt, H.V. Dedania and S.R. Patel
}

\begin{abstract}
Banach and Fréchet algebras with a Laurent series generator are investigated leading, via the discrete Beurling algebras, to functional analytic characterisations of the holomorphic function algebras on the annulus as well as the $C^{\infty}$-algebra on the unit circle.
\end{abstract}

\section{INTRODUCTION}

A linear associative unital algebra $A$ is a Fréchet algebra if $A$ is a complete metrisable topological algebra whose topology is defined by a sequence $\left(p_{k}\right)_{k \geqslant 1}$ of submultiplicative seminorms. Further, a Fréchet algebra $A$ has a Laurent series generator $x$ if

(a) $A$ is topologically generated by $\left\{x, x^{-1}\right\}$ for an invertible element $x$; and (b) for each $y \in A, y=\sum_{n \in \mathbb{Z}} \lambda_{n} x^{n}$, where $\sum_{n \in \mathbb{Z}}\left|\lambda_{n}\right| p_{k}\left(x^{n}\right)<\infty$ for all $k \in \mathbb{N}$.

A Fréchet algebra $A$ topologically generated by $\left\{x, x^{-1}\right\}$ has the unique expression property if each $y \in A$ has a unique expansion $y=\sum_{n \in \mathbb{Z}} \lambda_{n} x^{n}$.

A weight on $\mathbb{Z}$ is a strictly positive function $\omega: \mathbb{Z} \rightarrow(0, \infty)$ such that $\omega(m+n) \leqslant$ $\omega(m) \omega(n)$ for all $m, n$ in $\mathbb{Z}$. For $r>0$, let $\Gamma_{r}=\{z \in \mathbb{C}:|z|=r\}$. Let $\boldsymbol{\omega}=\left(\omega_{k}\right)_{k \geqslant 1}$ be an increasing sequence of weights on $\mathbb{Z}$. For an appropriate $r>0$, the associated Wiener Fréchet algebra $W\left(\Gamma_{r}, \omega\right)$ consists of continuous functions $f$ on $\Gamma_{r}$ satisfying $\|f\|_{\omega_{k}}:=\sum_{n \in \mathbb{Z}}\left|\widehat{f}_{r}(n)\right| \omega_{k}(n)<\infty$ for all $k \in \mathbb{N}$, where $f_{r}\left(e^{i \theta}\right)=f\left(r e^{i \theta}\right)(0 \leqslant \theta \leqslant 2 \pi)$ and $\hat{f}_{r}(n)$ is the $n^{\text {th }}$ Fourier coefficient of $f_{r}$. Then $W\left(\Gamma_{r}, \omega\right)$ is a Fréchet algebra with a Laurent series generator and with the topology defined by norms $\left\{\|\cdot\|_{\omega_{k}}: k \in \mathbb{N}\right\}$. For $0 \leqslant r_{2}<r_{1} \leqslant \infty$, let $H\left(\Gamma\left(r_{2}, r_{1}\right)\right)$ be the uniform Fréchet algebra consisting of functions holomorphic in the open annulus $\Gamma\left(r_{2}, r_{1}\right):=\left\{z \in \mathbb{C}: r_{2}<|z|<r_{1}\right\}$ and having the compact open topology. For $0<r_{2}<r_{1} \leqslant \infty$, let $\Gamma\left[r_{2}, r_{1}\right)=\left\{z \in \mathbb{C}: r_{2} \leqslant\right.$

Received 7th June, 2001

Thanks are due to Professor Ajit Iqbal Singh of Delhi University for pointing out an important gap in the first draft of the paper. The third author gratefully acknowledges the support of the Council of Scientific and Industrial Research (Government of India) in the form of CSIR Research Fellowship.

Copyright Clearance Centre, Inc. Serial-fee code: 0004-9727/02 \$A2.00+0.00. 
$\left.|z|<r_{1}\right\}$; the annuli $\Gamma\left(r_{2}, r_{1}\right]$ and $\Gamma\left[r_{2}, r_{1}\right]$ are analogously defined with appropriate $r_{1}$ and $r_{2}$. We shall write $\Gamma[r, r]=\Gamma_{r}$ and $\Gamma_{1}=\Gamma$ for simplicity. Let $H\left(\Gamma\left[r_{2}, r_{1}\right)\right)$ $:=\left\{f \in C\left(\Gamma\left[r_{2}, r_{1}\right)\right): f \in H\left(\Gamma\left(r_{2}, r_{1}\right)\right), f(z)=\sum_{n \in \mathbb{Z}} \lambda_{n} z^{n}\right.$ satisfying $q_{r}(f)<\infty$ for all $\left.0<r<r_{1}\right\}$, where $q_{r}(f)=\sum_{n \leqslant 0}\left|\lambda_{n}\right| r_{2}^{n}+\sum_{n>0}\left|\lambda_{n}\right| r^{n}$. Then $H\left(\Gamma\left[r_{2}, r_{1}\right)\right)$ is a Fréchet algebra with the topology defined by the norms $\left\{q_{r}: 0<r<r_{1}\right\}$. The Fréchet algebra $H\left(\Gamma\left(r_{2}, r_{1}\right]\right)$ is analogously defined. Let $H\left(\Gamma\left[r_{2}, r_{1}\right]\right)=\left\{f \in C\left(\Gamma\left[r_{2}, r_{1}\right]\right)\right.$ : $f \in H\left(\Gamma\left(r_{2}, r_{1}\right)\right), f(z)=\sum_{n \in \mathbb{Z}} \lambda_{n} z^{n}$ satisfying $\|f\|_{r_{2}, r_{1}}:=\sum_{n \leqslant 0}\left|\lambda_{n}\right| r_{2}^{n}+\sum_{n>0}\left|\lambda_{n}\right| r_{1}^{n}$ $<\infty\}$. Then $H\left(\Gamma\left[r_{2}, r_{1}\right]\right)$ is a Banach algebra with a Laurent series generator and with norm $\|\cdot\|_{r_{2}, r_{1}}$. Let $C^{\infty}(\Gamma)$ be the Fréchet algebra of all $C^{\infty}$-functions on $\Gamma$ with the topology of uniform convergence on $\Gamma$ of functions and all their derivatives.

We shall prove that a Laurent series generated Fréchet algebra having the unique expression property is isomorphic to the Beurling Fréchet algebra $\bigcap_{k=1}^{\infty} \ell^{1}\left(\mathbb{Z}, \omega_{k}\right)$ for an increasing sequence $\left(\omega_{k}\right)_{k \geqslant 1}$ of weights on $\mathbb{Z}$. This, in turn, will be used to prove the following main Theorem. We say that a Laurent series generator $x$ of a Fréchet algebra $\left(A,\left\{p_{k}\right\}\right)$ satisfies the condition $\left(^{*}\right)$ if

(i) there exists $M_{1}>0, \dot{M_{2}}>0$ and for each $k \in \mathbb{N}$, there exists $m_{k} \in \mathbb{N}$ such that

$$
M_{1}(1+|n|)^{m_{k}} \leqslant p_{k}\left(x^{n}\right) \leqslant M_{2}(1+|n|)^{m_{k}} \quad(n \in \mathbb{Z})
$$

and

(ii) there exists $n_{0} \in \mathbb{Z}$ such that $\left\{p_{k}\left(x^{n_{0}}\right): k \in \mathbb{N}\right\}$ is unbounded.

A uniform Fréchet algebra is a Fréchet algebra $\left(A,\left\{p_{k}\right\}\right)$ such that $p_{k}\left(a^{2}\right)$ $=p_{k}(a)^{2}$ for all $a \in A$ and $k \in \mathbb{N}$.

MaIn Theorem. Let $A$ be a Fréchet algebra with a Laurent series generator $x$ and having unique expression property. Then the following holds.

(a) If the spectrum $\operatorname{sp}_{A}(x)$ of the generator $x$ is open, then $A$ is homeomorphically isomorphic to $H\left(\Gamma\left(r_{2}, r_{1}\right)\right)$ for some $0 \leqslant r_{2}<r_{1} \leqslant \infty$. Further,

(i) $0<r_{2}$ if and only if zero belongs to the interior of $\mathbb{C} \backslash \operatorname{sp}_{A}(x)$; and

(ii) $r_{1}<\infty$ if and only if $\operatorname{sp}_{A}(x)$ is bounded.

(b) If the interior of $\operatorname{sp}_{A}(x)$ is empty, then there exists $r>0$ and a sequence $\boldsymbol{\omega}=\left(\omega_{k}\right)_{k \geqslant 1}$ of weights on $\mathbb{Z}$ such that $A$ is homeomorphically isomorphic to $W\left(\Gamma_{r}, \omega\right)$. Further, if the generator $x$ satisfies the condition $\left(^{*}\right)$, then 
$A$ is homeomorphically isomorphic to $C^{\infty}(\Gamma)$.

(c) If for each $k \in \mathbb{N}, p_{k}\left(x^{n}\right)=p_{k}(x)^{n}(n>0)$ and $p_{k}\left(x^{n}\right)=p_{k}\left(x^{-1}\right)^{-n}$ $(n<0)$, then $A$ is homeomorphically isomorphic to one of the Fréchet algebras $H\left(\Gamma\left(r_{2}, r_{1}\right)\right),\left(\Gamma\left[r_{2}, r_{1}\right)\right), H\left(\Gamma\left(r_{2}, r_{1}\right]\right)$ or $H\left(\Gamma\left[r_{2}, r_{1}\right]\right)$. Further, $\operatorname{sp}_{A}(x)$ is compact implies that $A$ is the Banach algebra $H\left(\Gamma\left[r_{2}, r_{1}\right]\right)$.

(d) - If $A$ is a uniform Fréchet algebra, then $A$ is homeomorphically isomorphic to $H\left(\Gamma\left(r_{2}, r_{1}\right)\right)$.

In Section 2, we discuss several relevant examples of Banach and Fréchet algebras having a Laurent series generator.

The proof of the main Theorem, presented in Section 3, is broken up into several lemmas of some independent interest. The paper is in the contemporary theme of exploiting power series ideas in Banach and Fréchet algebras. Though there are several functional analytic characterisations of holomorphic function algebras on simply connected planar domains (for example, [5, Theorems 12.3.2 and 13.1.7]), the case of annulus algebra appears to be treated for the first time in this paper.

\section{EXAMPLES}

Example 1. Beurling Fréchet algebras. Let $\omega$ be a weight on $\mathbb{Z}$. Let $\ell^{1}(\mathbb{Z}, \omega)$ consist of all formal Laurent series $f=\sum_{n \in \mathbb{Z}} \lambda_{n} X^{n}$ such that $\|f\|_{\omega}=\sum_{n \in \mathbb{Z}}\left|\lambda_{n}\right| \omega(n)<\infty$. Then $\left(\ell^{1}(\mathbb{Z}, \omega),\|\cdot\|_{\omega}\right)$ is a (Beurling) Banach algebra with the multiplication

$$
\left(\sum_{n \in \mathbb{Z}} \lambda_{n} X^{n}\right)\left(\sum_{n \in \mathbb{Z}} \mu_{n} X^{n}\right)=\sum_{n \in \mathbb{Z}}\left(\sum_{n=i+j} \lambda_{i} \cdot \mu_{j}\right) X^{n}
$$

and having Laurent series generator $X$ and Laurent series norm $\|\cdot\|_{\omega}$.

Let $\boldsymbol{\omega}=\left(\omega_{k}\right)_{k \geqslant 1}$ be a sequence (assumed increasing) of weights on $\mathbb{Z}$. Let $\ell^{1}(\mathbb{Z}, \omega)$ consist of all formal Laurent series $f:=\sum_{n \in \mathbb{Z}} \lambda_{n} X^{n}$ such that $\|f\|_{\omega_{k}}<\infty$ for all $k \in \mathbb{N}$. Then $\ell^{1}(\mathbb{Z}, \omega)$ is a Beurling Fréchet algebra having Laurent series generator $X$ in the topology defined by the family of norms $\|\cdot\|_{\omega_{k}}(k \in \mathbb{N})$. Define $\rho_{2, k}=\sup \left\{\omega_{k}(n)^{1 / n}\right.$ : $n \leqslant-1\}$ and $\rho_{1, k}=\inf \left\{\omega_{k}(n)^{1 / n}: n \geqslant 1\right\}$ for each $k$. Since $\left(\omega_{k}\right)$ is an increasing sequence, we have

$$
\cdots \leqslant \rho_{2,3} \leqslant \rho_{2,2} \leqslant \rho_{2,1} \leqslant \rho_{1,1} \leqslant \rho_{1,2} \leqslant \rho_{1,3} \leqslant \ldots
$$

The Fréchet algebra analogue of the classical Wiener Banach algebra is $W(\Gamma, \omega)$ consisting of continuous functions $f$ on $\Gamma$ having Fourier series $f \sim \sum_{n \in \mathbb{Z}} \widehat{f}(n) e^{i n \theta}$ which is $\boldsymbol{\omega}$-absolutely convergent in the sense that $\|f\|_{\omega_{k}}:=\sum_{n \in \mathbb{Z}}|\widehat{f}(n)| \omega_{k}(n)<\infty$ for all $k \in \mathbb{N}$. 
(Here we assume that $\omega_{1} \geqslant 1$.) With pointwise multiplication of functions, it is a Fréchet algebra having Laurent series generator $f(\theta)=e^{i \theta}$ in the topology defined by $\left\{\|\cdot\|_{\omega_{k}}: k \in \mathbb{N}\right\}$. The following is an important particular case of this.

Example 2. The Algebra $C^{\infty}(\Gamma)$. Let $C^{\infty}(\Gamma)$ consist of all $C^{\infty}$-functions on $\Gamma$. With the pointwise product, it is a Fréchet algebra with the Fréchet topology $\tau$ of uniform convergence on $\Gamma$ of all derivatives. It is defined by the norms $\|f\|_{C^{k}}$ $=\sum_{n=0}^{k}\left(\left\|f^{(n)}\right\|_{\infty}\right) / n !, 0 \leqslant k<\infty$, the Banach algebra norms on $C^{k}(\Gamma)$. For each $k \in \mathbb{N}$, let $\|f\|_{k}=\max _{0 \leqslant n \leqslant k}\left\|f^{(n)}\right\|_{\infty},\left(f \in C^{\infty}(\Gamma)\right)$. Then $\|\cdot\|_{k} \sim\|\cdot\|_{C^{k}}$. Let

$$
C^{k+}(\Gamma)=\left\{f \in C^{k}(\Gamma):\|f\|_{k+}:=\sum_{n \in \mathbb{Z}}|\widehat{f}(n)|\left|n^{k}\right|<\infty\right\}
$$

Then $C^{k+}(\Gamma)$ is a Laurent series generated Banach algebra with norm $\|\cdot\|_{k+}$ and having generator $e^{i \theta}$. Further,

$$
C^{k+1}(\Gamma) \subset C^{k+}(\Gamma) \subset C^{k}(\Gamma)
$$

and there is a constant $M>0$ such that $\|f\|_{C^{k}} \leqslant\|f\|_{k+} \leqslant M\|f\|_{C^{k+1}}$ for all $f$ belonging to $C^{k+1}(\Gamma)$. Hence

$$
C^{\infty}(\Gamma)=\bigcap_{k=0}^{\infty} C^{k}(\Gamma)=\bigcap_{k=0}^{\infty} C^{k+}(\Gamma)
$$

In view of the semisimplicity of $C^{\infty}(\Gamma)([1])$, the unique Fréchet algebra topology $\tau$ is also defined by the Laurent series seminorms $\left\{\|\cdot\|_{k+}: k \in \mathbb{N} \cup\{0\}\right\}$. This exhibits $C^{\infty}(\Gamma)$ as a Laurent series generated Fréchet algebra.

Example 3. The annulus Fréchet algebra. The following Fréchet algebras are defined in the introduction of this paper;

$$
\begin{array}{ll}
H\left(\Gamma\left(r_{2}, r_{1}\right)\right), & 0 \leqslant r_{2}<r_{1} \leqslant \infty \\
H\left(\Gamma\left[r_{2}, r_{1}\right)\right), & 0<r_{2}<r_{1} \leqslant \infty ; \\
H\left(\Gamma\left(r_{2}, r_{1}\right]\right), & 0 \leqslant r_{2}<r_{1}<\infty ; \\
H\left(\Gamma\left[r_{2}, r_{1}\right]\right), & 0<r_{2} \leqslant r_{1}<\infty .
\end{array}
$$

Each of these is a Fréchet algebra with a Laurent series generator $f(z)=z$, the last being a Banach algebra. For $0<r_{2}<r_{1}<\infty$, let $A\left(\Gamma\left[r_{2}, r_{1}\right]\right)$ be the uniform Banach algebra of all continuous functions $f$ on $\Gamma\left[r_{2}, r_{1}\right]$ which are holomorphic on 
$\Gamma\left(r_{2}, r_{1}\right)$ with the norm $\|f\|_{\infty}=\sup \left\{|f(z)|: z \in \Gamma\left[r_{2}, r_{1}\right]\right\}$. Now the inverse limit decompositions

$$
H\left(\Gamma\left(r_{2}, r_{1}\right)\right)=\lim _{n} A\left(\Gamma\left[r_{2}+\frac{1}{n}, r_{1}-\frac{1}{n}\right]\right)=\lim _{n} H\left(\Gamma\left[r_{2}+\frac{1}{n}, r_{1}-\frac{1}{n}\right]\right)
$$

(for sufficiently large $n$ ) shows that $H\left(\Gamma\left(r_{2}, r_{1}\right)\right)$ is a uniform Fréchet algebra with a Laurent series generator. This compares with Lemma 3.9 stating that an infinite dimensional Banach algebra with a Laurent series generator and having the unique expression property can never be a uniform Banach algebra.

For a weight $\omega$ on $\mathbb{Z}$, let $\rho_{2}:=\sup \left\{\omega(n)^{1 / n}: n<0\right\}$ and $\rho_{1}:=\inf \left\{\omega(n)^{1 / n}\right.$ : $n>0\}$. Let $A\left(\rho_{2}, \rho_{1}, \omega\right)$ consist of all continuous functions $f$ on $\Gamma\left[\rho_{2}, \rho_{1}\right]$ such that $f \in H\left(\Gamma\left(\rho_{2}, \rho_{1}\right)\right)$ and satisfying $\|f\|_{\omega}:=\sum_{n \in \mathbb{Z}}|\widehat{f}(n)| \omega(n)<\infty$. Then $A\left(\rho_{2}, \rho_{1}, \omega\right)$ is a Banach algebra with a Laurent series generator and with norm $\|\cdot\|_{\omega}$. For a sequence $\boldsymbol{\omega}=\left(\omega_{k}\right)$, let $\rho_{2}=\inf \left\{\rho_{2, k}: k \in \mathbb{N}\right\}, \rho_{1}=\sup \left\{\rho_{1, k}: k \in \mathbb{N}\right\}$, where $\rho_{2, k}$ and $\rho_{1, k}$ are as in Example 1. The Fréchet algebra $A\left(\rho_{2}, \rho_{1}, \omega\right)$ is an inverse limit of Banach algebras $A\left(\rho_{2, k}, \rho_{1, k}, \omega_{k}\right)$, and has a Laurent series generator.

\section{Proof of the Main Theorem}

LEMMA 3.1. Let $(A,\|\cdot\|)$ be a normed algebra with a Laurent series generator $x$. Assume that $\|\cdot\|$ is a Laurent series norm. Then the completion $(A,\|\cdot\|)^{\sim}$ is a Banach algebra with a Laurent series generator $x$.

Proof: Let $x$ be a Laurent series generator for $A$. Let $y \in A^{\sim}$. Choose a sequence

$$
y_{k}=\sum_{n \in \mathbb{Z}} \lambda_{k, n} x^{n},\left\|y_{k}\right\|=\sum_{n \in \mathbb{Z}}\left|\lambda_{k, n}\right|\left\|x^{n}\right\|
$$

in $A$ such that $\left\|y_{k}-y\right\| \rightarrow 0$. Now

$$
\sup _{n \in \mathbb{Z}}\left|\lambda_{k, n}-\lambda_{l, n}\right|\left\|x^{n}\right\| \leqslant \sum_{n \in \mathbb{Z}}\left|\lambda_{k, n}-\lambda_{l, n}\right|\left\|x^{n}\right\|=\left\|y_{k}-y_{1}\right\| \rightarrow 0
$$

shows that there exists a scalar sequence $\left(\lambda_{n}\right)_{n \in \mathbb{Z}}$ such that $\lambda_{k, n} x^{n} \rightarrow \lambda_{n} x^{n}$ uniformly over $n$. Then,

$$
\sum_{n \in \mathbb{Z}}\left|\lambda_{n}\right|\left\|x^{n}\right\|=\sum_{n \in \mathbb{Z}} \lim _{k}\left|\lambda_{k, n}\right|\left\|x^{n}\right\| \leqslant \underline{\lim }_{k} \sum_{n \in \mathbb{Z}}\left|\lambda_{k, n}\right|\left\|x^{n}\right\|=\underline{\lim }_{k}\left\|y_{k}\right\|<\infty
$$

showing that $\sum_{n \in \mathbb{Z}} \lambda_{n} x^{n} \in A^{\sim}$ and $\left\|y_{k}-\sum_{n \in \mathbb{Z}} \lambda_{n} x^{n}\right\| \rightarrow 0$. Hence $y=\sum_{n \in \mathbb{Z}} \lambda_{n} x^{n}$. 
This completes the proof.

LemmA 3.2. Let $(A,\|\cdot\|)$ be a Banach algebra which is Laurent series generated by $x$. Then $A$ has the unique expression property if and only if there exists a Laurent series norm $|\cdot|$ on $A$ equivalent to $\|\cdot\|$.

Proof: Let $|\cdot|$ be a Laurent series norm on $A$ equivalent to $\|\cdot\|$. Let $y \in A$ have expansions $y=\sum_{n \in \mathbb{Z}} \lambda_{n} x^{n}=\sum_{n \in \mathbb{Z}} \mu_{n} x^{n}$ such that $\sum_{n \in \mathbb{Z}}\left|\lambda_{n}\right|\left\|x^{n}\right\|<\infty$ and $\sum_{n \in \mathbb{Z}}\left|\mu_{n}\right|\left\|x^{n}\right\|<$ $\infty$. In view of the absolute convergence of these series, $0=\sum_{n \in \mathbb{Z}}\left(\lambda_{n}-\mu_{n}\right) x^{n}$; hence $0=|0|=\sum_{n \in \mathbb{Z}}\left|\lambda_{n}-\mu_{n}\right|\left|x^{n}\right|$. Since $x$ is invertible, $\left|x^{n}\right| \neq 0$ for all $n$. Thus $\lambda_{n}=\mu_{n}$ for all $n \in \mathbb{Z}$. This shows that $A$ has the unique expression property.

Conversely, assume that $A$ has the unique expression property. Given any $y=\sum_{n \in \mathbb{Z}} \lambda_{n} x^{n}$ in $A$ so that $\sum_{n \in \mathbb{Z}}\left|\lambda_{n}\right|\left\|x^{n}\right\|<\infty$, the unique expression property implies that $|y|:=\sum_{n \in \mathbb{Z}}\left|\lambda_{n}\right|\left\|x^{n}\right\|$ is well defined. We show that $|\cdot|$ defines the desired norm on $A$. Given $y=\sum_{n \in \mathbb{Z}} \lambda_{n} x^{n}, z=\sum_{n \in \mathbb{Z}} \mu_{n} x^{n}$ in $A$, so that $y+z=\sum_{n \in \mathbb{Z}}\left(\lambda_{n}+\mu_{n}\right) x^{n}$, we have

$$
|y+z|=\sum_{n \in \mathbb{Z}}\left|\lambda_{n}+\mu_{n}\right|\left\|x^{n}\right\| \leqslant \sum_{n \in \mathbb{Z}}\left|\lambda_{n}\right|\left\|x^{n}\right\|+\sum_{n \in \mathbb{Z}}\left|\mu_{n}\right|\left\|x^{n}\right\|=|y|+|z|,
$$

and $y z=\sum_{n \in \mathbb{Z}}\left(\sum_{m \in \mathbb{Z}} \lambda_{n-m} \mu_{m}\right) x^{n}$, with the result,

$$
|y z| \leqslant \sum_{n \in \mathbb{Z}} \sum_{m \in \mathbb{Z}}\left|\lambda_{n-m}\right|\left|\mu_{m}\right|\left\|x^{n-m}\right\|\left\|x^{m}\right\| \leqslant\left(\sum_{n \in \mathbb{Z}}\left|\lambda_{n}\right|\left\|x^{n}\right\|\right)\left(\sum_{n \in \mathbb{Z}}\left|\mu_{n}\right|\left\|x^{n}\right\|\right)=|y||z|
$$

showing that $|\cdot|$ is an algebra norm on $A$ satisfying $\|\cdot\| \leqslant|\cdot|$. We show that $(A,|\cdot|)$ is complete. Let $\left(y_{k}\right), y_{k}=\sum_{n \in \mathbb{Z}} \lambda_{k, n} x^{n}$ be a Cauchy sequence in $(A,|\cdot|)$. Then there exists $y \in A$ such that $\left\|y_{k}-y\right\| \rightarrow 0$. Thus

$$
\sup _{n \in \mathbb{Z}}\left|\lambda_{k, n}-\lambda_{l, n}\right|\left\|x^{n}\right\| \leqslant \sum_{n \in \mathbb{Z}}\left|\lambda_{k, n}-\lambda_{l, n}\right|\left\|x^{n}\right\|=\left|y_{k}-y_{l}\right| \rightarrow 0 .
$$

Hence for each $n \in \mathbb{Z}$, there exists $\lambda_{n} \in \mathbb{C}$ such that $\lambda_{n}\left\|x^{n}\right\|=\lim _{k} \lambda_{k, n}\left\|x^{n}\right\|$ uniformly over $n \in \mathbb{Z}$. Then $y=\sum_{n \in \mathbb{Z}} \lambda_{n} x^{n}$ and

$$
\begin{aligned}
\left|y_{k}-y\right|=\sum_{n \in \mathbb{Z}}\left|\lambda_{k, n}-\lambda_{n}\right|\left\|x^{n}\right\| & =\sum_{n \in \mathbb{Z}} \lim _{m}\left|\lambda_{k, n}-\lambda_{m, n}\right|\left\|x^{n}\right\| \\
& \leqslant \underline{\lim }_{m} \sum_{n \in \mathbb{Z}}\left|\lambda_{k, n}-\lambda_{m, n}\right|\left\|x^{n}\right\| \\
& =\underline{\lim }_{m}\left|y_{k}-y_{m}\right| \rightarrow 0 \text { as } k \rightarrow \infty .
\end{aligned}
$$


Thus $(A,|\cdot|)$ is a Banach algebra. By the Open Mapping Theorem, $|\cdot|$ and $\|\cdot\|$ are equivalent. This completes the proof.

LEMMA 3.3. Let $A$ be a Fréchet algebra with a Laurent series generator $x$. Let $p$ be a non-zero Laurent series seminorm on $A$. Then $p$ is a norm.

PROOF: Given that for any $y=\sum_{n \in \mathbb{Z}} \lambda_{n} x^{n}, p(y)=\sum_{n \in \mathbb{Z}}\left|\lambda_{n}\right| p\left(x^{n}\right)$. If $p\left(x^{m}\right)=0$ for some $m$, then $p(1) \leqslant p\left(x^{m}\right) p\left(x^{-m}\right)=0$; hence for all $n \in \mathbb{Z}, p\left(x^{n}\right) \leqslant p(1) p\left(x^{n}\right)=0$. Thus $p$ is identically zero, which is not possible. Thus $p\left(x^{n}\right) \neq 0$ for all $n \in \mathbb{Z}$. Now it follows that $p(y)=0$ implies $y=0$.

The following is a Fréchet algebra analogue of Lemma 3.2, which can be proved in the same way.

Lemma 3.4. Let $A$ be a Fréchet algebra having a Laurent series generator $x$. Then $A$ has the unique expression property if and only if the topology of $A$ is generated by a sequence of Laurent series norms.

LEMMA 3.5. Let $(A,\|\cdot\|)$ be a Banach algebra having a Laurent series generator $x$. Then there exists a continuous homomorphism from a Beurling algebra $\ell^{1}(\mathbb{Z}, \omega)$ onto $A$. Further, if $A$ has unique expression property, then $A \cong \ell^{1}(\mathbb{Z}, \omega)$.

Proof: Define $\omega(n)=\left\|x^{n}\right\|(n \in \mathbb{Z})$. Then $\omega(n+m) \leqslant \omega(n) \omega(m)$ for all $n$ and $m$ in $\mathbb{Z}$, and $x$ is not nilpotent since it is invertible. Hence $\omega$ is strictly positive, and thus $\omega$ is a weight on $\mathbb{Z}$. The map

$$
\Psi: \ell^{1}(\mathbb{Z}, \omega) \rightarrow A, \Psi\left(\sum_{n \in \mathbb{Z}} \lambda_{n} X^{n}\right)=\sum_{n \in \mathbb{Z}} \lambda_{n} x^{n}
$$

gives the desired surjective homomorphism. The assertion follows.

Now let $A$ and $\omega$ be as in Lemma 3.5 and let $A$ have the unique expression property. Then we have the spectral radius $r(x)=\lim _{n}\left\|x^{n}\right\|^{1 / n}=\inf _{n \geqslant 1}\left\|x^{n}\right\|^{1 / n}$ $=\inf _{n \geqslant 1} \omega(n)^{1 / n}=\rho_{1}$ say. Let $\rho_{2}=r\left(x^{-1}\right)^{-1}$. Then $\rho_{2}=\sup _{n \leqslant-1} \omega(n)^{1 / n}$. So $\rho_{2}=\inf \{|\lambda|: \lambda \in \operatorname{sp}(x)\} \leqslant \sup \{|\lambda|: \lambda \in \operatorname{sp}(x)\}=\rho_{1}$. Also, $0 \notin \operatorname{sp}(x)$ and $\operatorname{sp}(x)$ is a compact subset of $\mathbb{C}$. Hence, $\rho_{2}>0$. By [2, p. 118], the Gelfand space of $A$ is identified with $\Gamma\left[\rho_{2}, \rho_{1}\right] ;$ and the isomorphism $\ell^{1}(\mathbb{Z}, \omega) \rightarrow A\left(\rho_{2}, \rho_{1}, \omega\right), \sum_{n \in \mathbb{Z}} \lambda_{n} X^{n} \rightarrow \sum_{n \in \mathbb{Z}} \lambda_{n} z^{n}$ implies the following.

Corollary 3.6. Let $A$ be as in Lemma 3.5 and have the unique expression property. Let $\rho_{1}, \rho_{2}$ and $\omega$ be as above. Then $A$ is isomorphic to $A\left(\rho_{2}, \rho_{1}, \omega\right)$.

Next we discuss the Arens-Michael decomposition [3, pp. 88-90]. 
Lemma 3.7. Let $A$ be a Fréchet algebra generated by $x$ and $x^{-1}$. The following are equivalent.

(a) $A$ is Laurent series generated having Laurent series generator $x$ and having the unique expression property.

(b) There exists an inverse limit sequence

$$
A_{1} \stackrel{\pi_{1}}{\longleftarrow} A_{2} \stackrel{\pi_{2}}{\longleftarrow} A_{3} \stackrel{\pi_{3}}{\longleftarrow} A_{4} \stackrel{\pi_{4}}{\longleftarrow} \ldots
$$

of Laurent series generated Banach algebras $A_{k}$ with the unique expression property and having Laurent series generators $x_{k}$ such that $A=\lim _{k} A_{k}$.

Proof: Assume (a). By Lemma 3.4, $A$ is also a Fréchet algebra with a Laurent series generator whose Fréchet topology is given by an increasing sequence of Laurent series norms $\left(p_{k}\right)_{k \geqslant 1}$. The quotient map $\phi_{k}: A \rightarrow A / \operatorname{ker} p_{k}$ is continuous algebra homomorphism for each $k$, where $\operatorname{ker} p_{k}=\{0\}$. Hence that $A / \operatorname{ker} p_{k}=A$ and $\left(A, p_{k}\right)$ is a normed algebra with a Laurent series generator for each $k$. By Lemma 3.1, the completion $\left(A, p_{k}\right)^{\sim}=A_{k}$ is a Banach algebra with Laurent series generator $x_{k}$ for each $k$. By Arens-Michael decomposition, $A=\lim _{k} A_{k}$. This proves (b).

Conversely, assume (b). Let $y \in A, y=\left(y_{k}\right), y_{k}=\sum_{n \in \mathbb{Z}} \lambda_{k, n} x_{k}^{n}$. By Lemma 3.2, each $A_{k}$ is a Laurent series generated Banach algebra with a Laurent series norm. Thus $A=\lim _{k} A_{k}$ is a Fréchet algebra whose Fréchet topology is given by Laurent series norms $\left(p_{k}\right)_{k \geqslant 1}$, where $p_{k}(y)=\sum_{n \in \mathbb{Z}} \mid \lambda_{k, n}\left\|x_{k}^{n}\right\|_{k}[\mathbf{3}$, pp.86-87]. By Lemma 3.3, $\pi_{k}\left(x_{k+1}\right)=x_{k}$ implies $\lambda_{k+1, n}=\lambda_{k, n}=\lambda_{n}$ (say) for all $k$, and $n$. Hence

$$
\begin{aligned}
y=\left(y_{k}\right) & =\left(\sum_{n \in \mathbb{Z}} \lambda_{k, n} x_{k}^{n}\right) \\
& =\sum_{n \in \mathbb{Z}} \lambda_{n}\left(x_{k}\right)^{n} \\
& =\sum_{n \in \mathbb{Z}} \lambda_{n} x^{n} \text { where } x=\left(x_{k}\right) \in A,
\end{aligned}
$$

and

$$
\sum_{n \in \mathbb{Z}}\left|\lambda_{n}\right| p_{k}\left(x^{n}\right)=\sum_{n \in \mathbb{Z}}\left|\lambda_{k, n}\right|\left\|x_{k}^{n}\right\|_{k}<\infty \quad \forall k
$$

This proves (a).

Lemma 3.8. Let $A$ be a Fréchet algebra with a Laurent series generator and having the unique expression property. Then there exists an increasing sequence of weights $\boldsymbol{\omega}=\left(\omega_{k}\right)_{k \geqslant 1}$ on $\mathbb{Z}$ such that $A \cong \ell^{1}(\mathbb{Z}, \omega)=\bigcap_{k=1}^{\infty} \ell^{1}\left(\mathbb{Z}, \omega_{k}\right)$. 
ProOF: Let $x$ be a Laurent series generator for $A$. By Lemma 3.4, there exists an increasing sequence $\left(p_{k}\right)_{k \geqslant 1}$ of submultiplicative seminorms on $A$ such that $\left(p_{k}\right)_{k \geqslant 1}$ defines the topology of $A$ and each $p_{k}$ is a Laurent series seminorm. By Lemma 3.3, each $p_{k}$ is a norm. By Lemma 3.1, the completion $A_{k}:=\left(A, p_{k}\right)^{\sim}$ is Laurent series generated having Laurent series generator $x$. Since $p_{k} \leqslant p_{k+1}$, there exists continuous homomorphism $\pi_{k}: A_{k+1} \rightarrow A_{k}$ such that $\pi_{k \mid A}=$ id, the identity map on $A$. Thus

$$
A_{1} \stackrel{\pi_{1}}{\longleftarrow} A_{2} \stackrel{\pi_{2}}{\longleftarrow} A_{3} \stackrel{\pi_{3}}{\longleftarrow} A_{4} \stackrel{\pi_{4}}{\longleftarrow} \ldots
$$

is an inverse limit sequence; and by the general theory of Fréchet algebras, $A=\lim _{k} A_{k}$. By Lemma 3.5, there is a weight $\omega_{k}$ defined as $\omega_{k}(n)=p_{k}\left(x^{n}\right)$ for all $n \in \mathbb{Z}$ such that $A_{k} \cong \ell^{1}\left(\mathbb{Z}, \omega_{k}\right)$; the isomorphisms $\phi_{k}: A_{k} \rightarrow \ell^{1}\left(\mathbb{Z}, \omega_{k}\right)$ being $\phi_{k}=\Psi_{k}^{-1}$, (that is, $\left.\phi_{k}\left(\sum_{n \in \mathbb{Z}} \lambda_{n} x^{n}\right)=\sum_{n \in \mathbb{Z}} \lambda_{n} X^{n}\right)$, where the $\Psi_{k}$ are as in Lemma 3.5. We claim that $\pi_{k}$ is the identity map on $A_{k+1}$ for each $k$.

Indeed, for each $k$ and each $n, \omega_{k}(n)=p_{k}\left(x^{n}\right) \leqslant p_{k+1}\left(x^{n}\right)=\omega_{k+1}(n)$. Thus $\left(\omega_{k}\right)_{k \geqslant 1}$ is an increasing sequence and we have the following diagram,

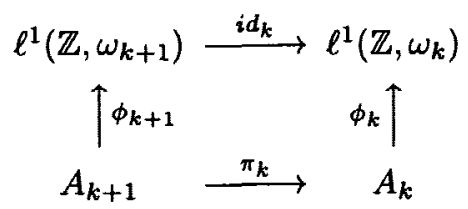

with $\left.\phi_{k}^{-1} \circ i d_{k} \circ \phi_{k+1}\right|_{A}=i d$. We show that this diagram is commutative, that is, $\phi_{k}^{-1} \circ \operatorname{id}_{k} \circ \phi_{k+1}=\pi_{k}$ on $A_{k+1}$. Let $y \in A_{k+1}$, say $y=\lim _{n} y_{n}$ in $p_{k+1}$ for some sequence $y_{n}=\sum_{j \in \mathbb{Z}} \lambda_{j}^{(n)} x^{j}$ (convergence in the topology of $A$ ). By the continuity of the homomorphism $\pi_{k}$, we have $\pi_{k}(y)=\lim _{n} \pi_{k}\left(y_{n}\right)=\lim _{n} y_{n}$ in $p_{k}$. Now by $\left(^{*}\right)$,

$$
\begin{aligned}
\pi_{k}(y) & =\lim _{n} y_{n} \text { in } p_{k} \\
& =\lim _{n}\left(\phi_{k}^{-1} \circ \mathrm{id}_{k} \circ \phi_{k+1}\right)\left(y_{n}\right) \\
& =\left(\phi_{k}^{-1} \circ \mathrm{id}_{k} \circ \phi_{k+1}\right)\left(\lim y_{n}\right) \text { in } p_{k+1} \\
& =\left(\phi_{k}^{-1} \circ \mathrm{id}_{k} \circ \phi_{k+1}\right)(y)
\end{aligned}
$$

showing $\pi_{k}=\phi_{k}^{-1} \circ \mathrm{id}_{k} \circ \phi_{k+1}$ on $A_{k+1}$. It follows that $\pi_{k}$ is one-one on $A_{k+1}$. Since $\pi_{k}=$ id on $A$, we obtain $\pi_{k}=$ id on $A_{k+1}$. Thus $A_{k+1} \subset A_{k}$ for all $k$, and

$$
A=\lim _{k} A_{k}=\bigcap_{k=1}^{\infty} A_{k} \cong \bigcap_{k=1}^{\infty} \ell^{1}\left(\mathbb{Z}, \omega_{k}\right) .
$$


This completes the proof of the lemma.

The following lemma exhibits an important difference between uniform Banach algebras and uniform Fréchet algebras at the level of algebras having a Laurent series generator. Recall that for $0<r_{2} \leqslant r_{1}<\infty$, the annulus uniform Banach algebra $A\left(\Gamma\left[r_{2}, r_{1}\right]\right)$ consists of functions $f \in C\left(\Gamma\left[r_{2}, r_{1}\right]\right)$ analytic in $\Gamma\left(r_{2}, r_{1}\right)$, with the supnorm on $\Gamma\left[r_{2}, r_{1}\right]$; in the case $r_{2}=r_{1}=r$ (say), $A\left(\Gamma\left[r_{2}, r_{1}\right]\right)=C\left(\Gamma_{r}\right)$.

Lemma 3.9. Let $(A,\|\cdot\|)$ be an infinite dimensional Banach algebra having a Laurent series generator and having the unique expression property. Then $A$ is not a uniform algebra.

Proof: By Lemma 3.5, there exists a weight $\omega$ on $\mathbb{Z}$ such that $A \cong \ell^{1}(\mathbb{Z}, \omega)$; and for any $f=\sum_{n \in \mathbb{Z}} \lambda_{n} x^{n}$ in $A$ having $\sum_{n \in \mathbb{Z}}\left|\lambda_{n}\right|\left\|x^{n}\right\|<\infty,\left\|\Psi^{-1}(f)\right\|_{\omega}=\sum_{n \in \mathbb{Z}}\left|\lambda_{n}\right| \omega(n)$. Without loss of generality, we can assume that $\omega(n) \geqslant 1$ for all $n \in \mathbb{Z}$. Indeed, let $z \in \mathbb{C}, \rho_{2} \leqslant|z| \leqslant \rho_{1}$; and let $\bar{\omega}(n)=\omega(n) /|z|^{n}(n \in \mathbb{Z})$. Then $\bar{\omega}$ is a weight on $\mathbb{Z}, \bar{\omega}(n) \geqslant 1$ for all $n \in \mathbb{Z}$, and the map $f \in \ell^{1}(\mathbb{Z}, \omega) \rightarrow z f \in \ell^{1}(\mathbb{Z}, \bar{\omega}),(z f)(n)=$ $z^{n} f(n)(n \in \mathbb{Z})$, establishes an isometric algebra isomorphism between $\ell^{1}(\mathbb{Z}, \omega)$ and $\ell^{1}(\mathbb{Z}, \bar{\omega})$. Thus we assume that $\omega(n) \geqslant 1$ for all $n \in \mathbb{Z}$. Hence, $\rho_{2} \leqslant 1 \leqslant \rho_{1}$. Notice that the Gelfand space $\Delta(A) \cong \Gamma\left[\rho_{2}, \rho_{1}\right]$ and $A \cong A\left(\rho_{2}, \rho_{1}, \omega\right)$. We identify isomorphic Banach algebras.

Now suppose that $A$ is a uniform Banach algebra. Then $A$ is complete in the spectral radius norm $r_{A}(f):=\sup \left\{|f(z)|: z \in \Gamma\left[\rho_{2}, \rho_{1}\right]\right\}=|f|$ say for $f \in A$. Since $|\cdot|$ is a uniform norm satisfying $\left|f^{n}\right|=|f|^{n}(f \in A, n \in \mathbb{Z})$ and is equivalent to $\|\cdot\|$, it follows that for any $f=\sum_{n \in \mathbb{Z}} \lambda_{n} x^{n}$ in $A, \sum_{n=-\infty}^{0}\left|\lambda_{n}\right| \rho_{2}^{n}+\sum_{n=0}^{\infty}\left|\lambda_{n}\right| \rho_{1}^{n}<\infty$.

CASE I. Let $\rho_{2}<\rho_{1}$. Then by the above, $A=H\left(\Gamma\left[\rho_{2}, \rho_{1}\right]\right)$. On the other hand, by the completeness of $(A,|\cdot|), A=A\left(\Gamma\left[\rho_{2}, \rho_{1}\right]\right)$. This yields a contradiction. Indeed, by a classical result $[4, \mathrm{p}, 264]$, there exists a function $f$ continuous on the closed unit disc $D=\{z \in \mathbb{C}:|z| \leqslant 1\}$ which is analytic in the interior of $D$ and the $m^{\text {th }}$ partial sum of whose Taylor series satisfies $\left\|s_{m}(f)\right\|_{\infty} \geqslant\left|s_{m}(f)(1)\right|=O(\log m),\|\cdot\|_{\infty}$ being the supremum norm on $D$. Thus the Taylor series of $f$ fails to be absolutely convergent on $\Gamma$. Then the function $g(z)=f\left(z / \rho_{1}\right)$ is in $A\left(\Gamma\left[\rho_{2}, \rho_{1}\right]\right)$ but fails to be in $H\left(\Gamma\left[\rho_{2}, \rho_{1}\right]\right)$. CASE II. Let $\rho_{2}=1=\rho_{1}$. In this case $A \cong W(\Gamma, \omega)$, the weighted Wiener algebra. Since $\Delta(A)=\Gamma,|f|=\sup \{|f(z)|: z \in \Gamma\}$. Then the completeness of $(A,|\cdot|)$ contradicts the classical fact that there exists a continuous function on $\Gamma$ whose Fourier series fails to be absolutely convergent. This completes the proof of the lemma.

3.10 Proof of The Main Theorem. Let $A$ be a Fréchet algebra with a Laurent series generator $x$ and having the unique expression property. Then by Lemma 3.4, the 
topology on $A$ is generated by a sequence $\left(p_{k}\right)$ of Laurent series seminorms. By Lemma 3.3 , each $p_{k}$ is in fact a Laurent series norm. We may assume that $p_{1} \leqslant p_{2} \leqslant p_{3} \leqslant \ldots$. By Lemma 3.8, $A \cong \ell^{1}(\mathbb{Z}, \boldsymbol{\omega})=\bigcap_{k=1}^{\infty} \ell^{1}\left(\mathbb{Z}, \omega_{k}\right)$, where $\omega_{k}(n)=p_{k}\left(x^{n}\right)(n \in \mathbb{Z})$ for each $k \in \mathbb{N}$. Then

$$
\Delta(A)=\bigcup_{k=1}^{\infty} \Delta\left(A_{k}\right) \cong \bigcup_{k=1}^{\infty} \Delta\left(\ell^{1}\left(\mathbb{Z}, \omega_{k}\right)\right)=\bigcup_{k=1}^{\infty} \Gamma\left[\rho_{2, k}, \rho_{1, k}\right]
$$

and

$$
\operatorname{sp}_{A}(x)=\bigcup_{k=1}^{\infty} \operatorname{sp}_{A_{k}}(x)=\bigcup_{k=1}^{\infty} \operatorname{sp}_{\ell^{1}\left(\mathbb{Z}, \omega_{k}\right)}(x)=\bigcup_{k=1}^{\infty} \Gamma\left[\rho_{2, k}, \rho_{1, k}\right] .
$$

Now define $\rho_{2}=\inf \left\{\rho_{2, k}: k \in \mathbb{N}\right\}$ and $\rho_{1}=\sup \left\{\rho_{1, k}: k \in \mathbb{N}\right\}$. Then $0 \leqslant \rho_{2} \leqslant \rho_{1} \leqslant \infty$. Sinc the generator $x$ is invertible, it is not true that $\rho_{2}=\rho_{1}=0$. Similarly it is not true that $\rho_{2}=\rho_{1}=\infty$. Hence we have either $0 \leqslant \rho_{2}<\rho_{1} \leqslant \infty$ or $0<\rho_{2}=\rho_{1}<\infty$.

(a) Suppose that $\operatorname{sp}_{A}(x)$ is open. Then by definitions of $\rho_{2}$ and $\rho_{1}$ and equations (1) and (2) above, we have $\Delta(A) \cong \Gamma\left(\rho_{2}, \rho_{1}\right)=\mathrm{sp}_{A}(x)$. Now define the Gelfand transform $F: A \rightarrow H\left(\Gamma\left(\rho_{2}, \rho_{1}\right)\right)$ as $F(y)=\widehat{y}$, where for $y=\sum_{n \in \mathbb{Z}} \lambda_{n} x^{n} \in A$, we have $\widehat{y}(z)=\sum_{n \in \mathbb{Z}} \lambda_{n} z^{n}\left(z \in \Gamma\left(\rho_{2}, \rho_{1}\right)\right)$. It is clear that the map $F$ is one to one and continuous. To prove that it is onto, let $f \in H\left(\Gamma\left(\rho_{2}, \rho_{1}\right)\right)$ with $f(z)=\sum_{n \in \mathbb{Z}} \lambda_{n} z^{n}$ $\left(z \in \Gamma\left(\rho_{2}, \rho_{1}\right)\right)$. Then the series is uniformly and absolutely convergent on each compact subset of $\Gamma\left(\rho_{2}, \rho_{1}\right)$. Fix $k \in \mathbb{N}$. Then $\rho_{2}<\rho_{2, k} \leqslant \rho_{1, k}<\rho_{1}$. So choose $\rho_{2}<r_{2}$ $<\rho_{2, k} \leqslant \rho_{1, k}<r_{1}<\rho_{1}$. Then $\sum_{n<0}\left|\lambda_{n}\right| r_{2}^{n}+\sum_{n \geqslant 0}\left|\lambda_{n}\right| r_{1}^{n}<\infty$. Choose $n_{0} \in \mathbb{N}$ such that $r_{2}<\omega_{k}(n)^{1 / n}$ when $n \leqslant-n_{0}$ and $\omega_{k}(n)^{1 / n}<r_{1}$ when $n \geqslant n_{0}$. This implies

$$
\sum_{n \leqslant-n_{0}}\left|\lambda_{n}\right| \omega_{k}(n)+\sum_{n \geqslant n_{0}}\left|\lambda_{n}\right| \omega_{k}(n)<\sum_{n \leqslant-n_{0}}\left|\lambda_{n}\right| r_{2}^{n}+\sum_{n \geqslant n_{0}}\left|\lambda_{n}\right| r_{1}^{n}<\infty,
$$

hence $\sum_{n \in \mathbb{Z}}\left|\lambda_{n}\right| \omega_{k}(n)<\infty$, and so $\sum_{n \in \mathbb{Z}} \lambda_{n} X^{n} \in \ell^{1}\left(\mathbb{Z}, \omega_{k}\right) \cong A_{k}$. This is true for each $k \in \mathbb{N}$. Thus $y=\sum_{n \in \mathbb{Z}} \lambda_{n} x^{n} \in A$ and $F(y)=\widehat{y}=f$. So $F$ is onto. This proves that $A \cong H\left(\Gamma\left(\rho_{2}, \rho_{1}\right)\right) .^{\prime}$ In this case, it is now clear that $0<\rho_{2}$ if and only if the zero is in the interior of $\mathbb{C} \backslash \mathrm{sp}_{A}(x)$; and that $\rho_{1}<\infty$ if and only if $\operatorname{sp}_{A}(x)$ is bounded.

(b) Assume that the interior of $\operatorname{sp}_{A}(x)$ is empty. Then it follows from equation (2) that $\bigcup_{k=1}^{\infty} \Gamma\left[\rho_{2, k}, \rho_{1, k}\right]$ is a circle (say $\Gamma_{\rho}$ ). Then we have $\rho_{2, k}=\rho_{1, k}=\rho_{2}=\rho_{1}=\rho$. So for each $k$, we have $\Delta\left(A_{k}\right)=\Delta\left(\ell^{1}\left(\mathbb{Z}, \omega_{k}\right)\right)=\{z \in \mathbb{C}:|z|=\rho\}=\Gamma_{\rho}$. Also it 
is clear that we have $\ell^{1}\left(\mathbb{Z}, \omega_{k}\right) \cong\left\{f \in C\left(\Gamma_{\rho}\right): f \in W\left(\Gamma_{\rho}, \omega_{k}\right)\right\}=\left\{f \in C\left(\Gamma_{\rho}\right)\right.$ : $\left.\sum_{n \in \mathbb{Z}}\left|\hat{f}_{\rho}(n)\right| \omega_{k}(n)<\infty\right\}=W\left(\Gamma_{\rho}, \omega_{k}\right)$. Hence $A=\bigcap_{k=1}^{\infty} \ell^{1}\left(\mathbb{Z}, \omega_{k}\right) \cong \bigcap_{k=1}^{\infty} W\left(\Gamma_{\rho}, \omega_{k}\right)$ $=W\left(\Gamma_{\rho}, \omega\right)$, where $\omega=\left(\omega_{k}\right)$.

Further, assume that the condition $\left(^{*}\right)$ is satisfied. Then condition (i) of $\left(^{*}\right)$ implies that $\rho=1$. For for each $k, \rho_{1, k}=\rho_{2, k}=1$. Thus $y=\sum_{n \in \mathbb{Z}} \lambda_{n} x^{n} \in A$ if and only if $\widehat{y}=f$ for some $f \in C(\Gamma)$ satisfying $\sum_{n \in \mathbb{Z}}|\widehat{f}(n)| \omega_{k}(n)<\infty$ (for all $k \in \mathbb{N}$ ). Condition (ii) of $\left(^{*}\right)$ implies that for some $m \in \mathbb{Z},\left(p_{k}\left(x^{m}\right)\right)_{k \geqslant 1}$ is unbounded. By passing to a subsequence, if necessary, we assume that $p_{k}\left(x^{m}\right) \rightarrow \infty$ as $k \rightarrow \infty$. Thus, the $\left(m_{k}\right)$ of condition (i) of (*) satisfies $m_{k} \rightarrow \infty$ as $k \rightarrow \infty$. Without loss of generality, assume that $m_{k}=k$. Thus, by using condition (i) of $\left(^{*}\right), y \in A$ if and only if $\hat{y}=f$ for some $f \in C(\Gamma)$ and $\sum_{n \in \mathbb{Z}}|\widehat{f}(n)|(a+|n|)^{k}<\infty$ (for all $k \in \mathbb{N}$ ). In view of Example 2, this happens if and only if $f \in C^{\infty}(\Gamma)$.

(c) Suppose that for each $k \in \mathbb{N}, p_{k}\left(x^{n}\right)=p_{k}(x)^{n}(n>0)$ and $p_{k}\left(x^{n}\right)$ $=p_{k}\left(x^{-1}\right)^{-n}(n<0)$. Then for each $k \in \mathbb{N}$, we have $\omega_{k}(n)=\omega_{k}(1)^{n}(n>0)$ and $\omega_{k}(n)=\omega_{k}(-1)^{-n}(n<0)$, where $\rho_{2, k}=p_{k}\left(x^{-1}\right)^{-1}=\omega_{k}(-1)^{-1}, \rho_{1, k}=p_{k}(x)$ $=\omega_{k}(1)$. So the Gelfand space $\Delta(A)$ is topologically homeomorphic to any one of the following annuli: $\Gamma\left(\rho_{2}, \rho_{1}\right), \Gamma\left(\rho_{2}, \rho_{1}\right], \Gamma\left[\rho_{2}, \rho_{1}\right)$ or $\Gamma\left[\rho_{2}, \rho_{1}\right]$. Suppose that $\Delta(A)$ $\cong \Gamma\left(\rho_{2}, \rho_{1}\right]$. Then it is clear that $\rho_{1}<\infty$. In order to prove that $A \cong H\left(\Gamma\left(\rho_{2}, \rho_{1}\right]\right)$, it is enough to prove that for each $f \in H\left(\Gamma\left(\rho_{2}, \rho_{1}\right]\right)$, there exists $y \in A$ such that $\widehat{y}=f$. So fix $f \in H\left(\Gamma\left(\rho_{2}, \rho_{1}\right]\right)$. Then we have $f(z)=\sum_{n \in \mathbb{Z}} \lambda_{n} z^{n}\left(z \in \Gamma\left(\rho_{2}, \rho_{1}\right]\right)$ such that $q_{r}(f)=\sum_{n<0}\left|\lambda_{n}\right| r^{n}+\sum_{n \geqslant 0}\left|\lambda_{n}\right| \rho_{1}^{n}<\infty$ (for all $\rho_{2}<r$ ). Fix $k \in \mathbb{N}$. Then $\rho_{2}<\rho_{2, k} \leqslant \rho_{1, k} \leqslant \rho_{1}$. This implies that $\sum_{n<0}\left|\lambda_{n}\right| \rho_{2, k}^{n}+\sum_{n \geqslant 0}\left|\lambda_{n}\right| \rho_{1, k}^{n}<\infty$, that is, $\sum_{n \in \mathbb{Z}}\left|\lambda_{n}\right| \omega_{k}(n)<\infty$. Thus $\sum_{n \in \mathbb{Z}} \lambda_{n} X^{n} \in \ell^{1}\left(\mathbb{Z}, \omega_{k}\right)$, that is, $y=\sum_{n \in \mathbb{Z}} \lambda_{n} x^{n} \in A_{k}$. This is true for each $k \in \mathbb{N}$. Hence $y \in A$ and $\hat{y}=f$. The other cases are similar.

Further, suppose that $\operatorname{sp}_{A}(x)$ is compact. Then we have $\rho_{2}>0, \rho_{1}<\infty$ and $\operatorname{sp}_{A}(x)=\Gamma\left[\rho_{2}, \rho_{1}\right]$. This implies that there exists $m \in \mathbb{N}$ such that $\rho_{2}=\rho_{2, m}$ and $\rho_{1}=\rho_{1, m}$. Define $\omega(n)=\omega_{m}(1)^{n}(n>0)$ and $\omega(n)=\omega_{m}(-1)^{-n}(n<0)$, where $\rho_{2}=\omega_{m}(-1)^{-1}, \rho_{1}=\omega_{m}(1)$. Then it is clear that $A \cong H\left(\Gamma\left[\rho_{2}, \rho_{1}\right]\right) \cong \ell^{1}(\mathbb{Z}, \omega)$ which is a Banach algebra.

(d) Assume that $A$ is a uniform Fréchet algebra. Since $A$ has a Laurent series generator, it can not be a uniform Banach algebra by Lemma 3.9. So the sequence $\left(p_{k}\right)_{k \geqslant 1}$ is infinite. Since $p_{k}\left(y^{2}\right)=p_{k}(y)^{2}(y \in A)$, it is easy to see that $\left(p_{k}\right)_{k \geqslant 1}$ satisfies the hypothesis of (c) above. Since none of the Fréchet algebras $H\left(\Gamma\left[r_{2}, r_{1}\right]\right)$, 
$H\left(\Gamma\left[r_{2}, r_{1}\right)\right), H\left(\Gamma\left(r_{2}, r_{1}\right]\right)$ is a uniform Fréchet algebra, $A$ is homeomorphically isomorphic to $H\left(\Gamma\left(r_{2}, r_{1}\right)\right)$.

REMARK 3.11. The following sequences $\omega=\left(\omega_{k}\right)_{k \geqslant 1}$ exhibits the different cases considered in the statement of the main Theorem.

(1) $\omega_{k}(n)=(k+1)^{|n|}(n \in \mathbb{Z}) ; \ell^{1}(\mathbb{Z}, \omega) \cong H(\Gamma(0, \infty))$.

(2) $\omega_{k}(n)=1(n \geqslant 0)$ and $\omega_{k}(n)=(k+1)^{-n}(n<0) ; \ell^{1}(\mathbb{Z}, \omega) \cong H(\Gamma(0,1])$.

(3) $\omega_{k}(n)=(k+1)^{n}(n \geqslant 0)$ and $\omega_{k}(n)=1(n<0) ; \ell^{1}(\mathbb{Z}, \omega) \cong H(\Gamma[1, \infty))$.

(4) $\omega_{k}(n)=(1+|n|)^{k}(n \in \mathbb{Z}) ; \ell^{1}(\mathbb{Z}, \omega) \cong C^{\infty}(\Gamma)$.

\section{REFERENCES}

[1] R.L. Carpenter, 'Uniqueness of topology for commutative semisimple $F$-algebras', Proc. Amer. Math. Soc. (1971), 113-117.

[2] I. Gelfand, D. Raikov and G. Shilov, Commutative normed rings (Chelsea Publ. Co., Bronx, NY, 1964).

[3] H. Goldmann, Uniform Fréchet algebras, North Holland Mathematics Studies 162 (North Holland Publ. Co., Amsterdam, 1990).

[4] R.J. Loy, 'Banach algebras of power series', J. Austral. Math. Soc. 17 (1974), 263-273.

[5] D. Wang and S. Watson, 'A characterization of the algebra of holomorphic functions on a simply connected domain', Internat. J. Math. and Math. Sci. 12 (1989), 65-68.

Department of Mathematics

Sardar Patel University

Vallabh Vidyanagar 388120

Gujarat

India

e-mail: sjb@spu.ernet.in

haresh@spu.ernet.in

coolpatel@spu.ernet.in 